\title{
New Insights in the Amyloid-Beta Interaction with Mitochondria
}

\author{
Carlos Spuch, Saida Ortolano, and Carmen Navarro \\ Department of Pathology and Neuropathology, Hospital of Meixoeiro, University Hospital of Vigo, Meixoeiro s/n, 36215 Vigo, Spain
}

Correspondence should be addressed to Carlos Spuch, carlos.spuch@gmail.com

Received 28 November 2011; Accepted 11 January 2012

Academic Editor: Jan Vijg

Copyright ( 12012 Carlos Spuch et al. This is an open access article distributed under the Creative Commons Attribution License, which permits unrestricted use, distribution, and reproduction in any medium, provided the original work is properly cited.

\begin{abstract}
Biochemical and morphological alterations of mitochondria may play an important role in the pathogenesis of Alzheimer's disease (AD). Particularly, mitochondrial dysfunction is a hallmark of amyloid-beta-induced neuronal toxicity in Alzheimer's disease. The recent emphasis on the intracellular biology of amyloid-beta and its precursor protein (APP) has led researchers to consider the possibility that mitochondria-associated and mitochondrial amyloid-beta may directly cause neurotoxicity. Both proteins are known to localize to mitochondrial membranes, block the transport of nuclear-encoded mitochondrial proteins to mitochondria, interact with mitochondrial proteins, disrupt the electron transport chain, increase reactive oxygen species production, cause mitochondrial damage, and prevent neurons from functioning normally. In this paper, we will outline current knowledge of the intracellular localization of amyloid-beta. Moreover, we summarize evidence from $\mathrm{AD}$ postmortem brain as well as animal $\mathrm{AD}$ models showing that amyloid-beta triggers mitochondrial dysfunction through a number of pathways such as impairment of oxidative phosphorylation, elevation of reactive oxygen species production, alteration of mitochondrial dynamics, and interaction with mitochondrial proteins. Thus, this paper supports the Alzheimer cascade mitochondrial hypothesis such as the most important early events in this disease, and probably one of the future strategies on the therapy of this neurodegenerative disease.
\end{abstract}

\section{Introduction}

Each year, over 10 million people globally suffer from neurodegenerative diseases. This figure is expected to grow by $20 \%$ over the next decade as the aging population increases and lives longer. This disease group is the fourth biggest killer in the developed world after heart diseases, cancer, and stroke [1]. The most common neurodegenerative diseases are $\mathrm{AD}$, Parkinson disease, Lewy body dementia, frontotemporal dementia, and amyotrophic lateral sclerosis [2]. The most widely recognized is $\mathrm{AD}$, which is among the principal debilitating conditions of the current century. Approximately 24 million people worldwide suffer from dementia, $60 \%$ of cases being due to $\mathrm{AD}$, which occurs in $1 \%$ of individuals aged 50 to 70 years old and dramatically increases to $50 \%$ of those over 70 years old [3]. Dramatically, these numbers are estimated to increase to 15 million in the next 40 years [4].

From the neuropathological point of view, AD is characterized by selective neuronal loss, marked synaptic alteration, morphological mitochondrial abnormalities, and Tau pathology. The histological hallmark lesions of $\mathrm{AD}$ are characterized by senile plaques and cerebrovascular deposits. The extracellular amyloid plaques mainly composed of amyloid-beta and intracellular neurofibrillary tangles built up of hyperphosphorylated tau, although the molecular mechanisms underlying the disease are still unknown and there is still no cure. Many lines of evidence suggest that oxidative stress is one of the earliest changes and plays an important role in the pathological process in $\mathrm{AD}$, and more recently, energy deficiency and mitochondrial dysfunction have been recognized as a prominent, early event in $\mathrm{AD}$ [111]. Oxidative stress plays a critical role in the pathogenesis of $\mathrm{AD}$ and is intimately linked to aging, the best established risk factor for $\mathrm{AD}$. Increased oxidative stress levels have been found in the brains of patients with $A D$ in Sweden and early in Tg2576 APP transgenic mice [12, 13]. Recently, we described that PLA2G3 gene silencing produced a marked inhibition of the free radical-generating xanthine/xanthine oxidase- (X-XOD-) system-induced cell death, and that PLA2G3 polymorphisms are associated with $\mathrm{AD}$ in a Spanish case-control sample [14]. In previous studies with choroid plexus homogenates with different cases with $\mathrm{AD}$ in the 
different stages (I/0, III-IV, and V-VI), we demonstrated that in $\mathrm{AD}$ patients, amyloid-beta peptide also accumulates in choroid plexus [15], there is an oxidation of carboxymethyllysine (CML), and $\mathrm{N}$-carboxyethyl-lysine (CEL) may result in impaired protein interactions, protein folding, and protein kinase activity; abnormal function of endothelial and vascular smooth muscle cells; impaired HDL-cholesterol metabolism in the choroid plexus in advanced stages of AD [16].

\section{Mitochondria}

Mitochondria are found in virtually all eukaryotic cells and function to generate cellular energy in the form of adenosine triphosphate (ATP) by oxidative phosphorylation and are thought to be derived evolutionarily from the fusion of prokaryotic and eukaryotic organisms [17]. They are also involved in regulation of cell death via apoptosis, in the control of cell division and growth, in calcium homeostasis, haem biosynthesis, and in the formation and export of ironsulphur clusters.

Mitochondria are composed of a double lipid membrane which structures four compartments, distinct by composition and function (Figure 1). The porous outer membrane encompasses the whole organelle. It contains many proteins like import complexes and voltage-dependent anion channels responsible for the free passage of lowmolecular-weight substances (up to $5000 \mathrm{Da}$ ) between the cytoplasm and the intermembrane space which represents a reservoir of protons establishing a proton electrochemical gradient across inner mitochondrial membrane that is needed for the production of ATP via ATPase (complex $\mathrm{V})$. Intermembrane space contains proapoptotic proteins like cytochrome c, Smac/Diablo, EndoG, and Htra2/Omi. In contrast to the permeable outer membrane, the inner mitochondrial membrane, rich in cardiolipin, provides a highly efficient barrier to the flow of small molecules and ions, including protons. This membrane is invaginated into numerous cristae increasing cell surface area. It houses the respiratory enzymes of the electron transport chain, the cofactor coenzyme $\mathrm{Q}$, and many mitochondrial carriers. In the matrix, different metabolic pathways take place including the tricarboxylic or Krebs cycle [18].

Mitochondria are unique amongst cellular organelles in that they have their own, circular, double-stranded DNA (mtDNA) which is inherited almost exclusively down the maternal line and codes for 37 mitochondrial genes, 13 of which translate to proteins involved in oxidative phosphorylation [18, 19]. The remaining genes encode transfer (22 genes) and ribosomal (2 genes) RNA allowing the mitochondria to generate their own proteins. Although mitochondria have the ability to produce proteins, the vast majority of proteins that function within the mitochondria, including those involved in DNA transcription, translation, and repair, are encoded by nuclear DNA and are transported into the mitochondria from the cytosol. As mtDNA is located in the mitochondria in close proximity to the electron

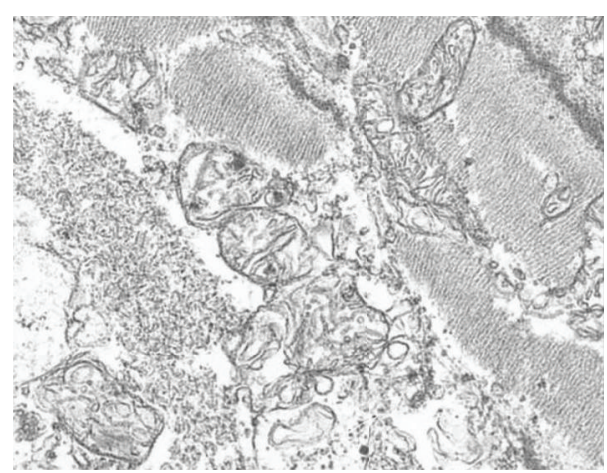

FIGURE 1: Representative electromicrographs of mitochondrial ultrastructure. Scale bar $200 \mathrm{~nm}$.

transport chain, it is more susceptible to damage from free radicals generated during oxidative phosphorylation [20].

Mitochondria generate energy by two closely coordinated metabolic processes: Krebs cycle and the oxidative phosphorylation (OXPHOS). OXPHOS is made up of the electron transport chain assembled in four enzymes (complex I to IV) as well as the F1F0-ATP synthase (complex V). Complexes I, III, and IV are located in inner mitochondrial membrane as integral proteins, whereas complex II is attached to the inner surface of this membrane. The function of the chain is to generate cellular energy in the form of ATP. These five enzymes of the complex are connected functionally by mobile electron acceptors and donors: ubiquinone and cytochrome c. Electrons from NADH and $\mathrm{FADH}_{2}$ are fed into complexes I and II, respectively. Ubiquinone $\mathrm{Q}$ carries electrons from both complexes to complex II, and cytochrome $\mathrm{c}$ does it from complex III to IV reducing molecular oxygen to water. As electrons are transferred along electron transport chain, a fixed number of protons are pumped from the matrix into inner membrane space establishing a electrochemical gradient characterized with a specific electrical potential. The redox energy drives the synthesis of ATP from ADP as protons are transported back from inner membrane space into the matrix via complex $\mathrm{V}$.

\section{Amyloid-Beta in the Cytosol}

Accumulation of amyloid-beta in $\mathrm{AD}$ brains is thought to underlie neuronal dysfunction and memory loss, being centrally implicated in AD pathogenesis. Moreover, previous to mitochondrial accumulation of amyloid-beta showed in $\mathrm{AD}$ patient and $\mathrm{AD}$ transgenic mouse brain, the toxic amyloid-beta species has to be accumulated in the cytosol of the cells. Many studies showed amyloid-beta interaction with different receptors in the cellular membrane of the vasculature, neurons, oligodendrocytes, and glial cells where it is transported from cell surface into endosomal and lysosomal compartments [21-23]. The aberrant signalling of these receptors in $\mathrm{AD}$ triggered an abnormal accumulation of amyloid-beta into cytosol-inducing cellular stress underlies to neuronal dysfunction and dementia. 
It is described by our group and others that these receptors can be megalin, also known as low-density lipoproteinrelated protein-2 (LRP2) [24], LRP-1 [22], or RAGE (receptors for advanced glycation end products) [25]. The interaction of these receptors with amyloid-beta in neurons, microglia, and vascular cells accelerates and amplifies deleterious effects on neuronal and synaptic functions. These findings are further in line with the recently proposed hypothesis of an intracellular amyloid-beta toxicity cascade which suggests that the toxic amyloid-beta species intervening in molecular and biochemical abnormalities may be intracellular soluble aggregates instead of extracellular, insoluble plaques. There are many studies proposing that megalin- and/or RAGE-dependent signalling are involved in the regulation of amyloid-beta clearance and probably may contribute to amyloid pathology and cognitive dysfunction observed in the AD patients and AD mouse model.

\section{Amyloid-Beta and Mitochondria}

Studies of postmortem brains from AD patients and transgenic mouse models of $\mathrm{AD}$ suggest that oxidative damage, induced by amyloid-beta, is associated with mitochondria early in AD progression. Amyloid-beta and APP protein are known to localize to mitochondrial membranes, block the transport of nuclear-encoded mitochondrial proteins to mitochondria, interact with mitochondrial proteins, disrupt the electron transport chain, increase reactive oxygen species (ROS) production, cause mitochondrial damage, and prevent neurons from functioning normally. Recent scientific research has identified multiple mechanisms of amyloid-beta interaction with mitochondria at different mitochondrial compartments: the outer mitochondrial membrane, intermembrane space, inner mitochondrial membrane, and the matrix. It is well known that the involvement of amyloidbeta-induced mitochondrial dysfunction in $\mathrm{AD}$ pathogenesis, a vicious cycle as well as several vicious circles within the cycle, each accelerating the other, can be drawn emphasizing the Alzheimer mitochondrial cascade hypothesis.

The brain is vulnerable to oxidative stress owing to its high lipid content, its relatively high oxygen metabolism, and its low levels of antioxidant defenses [26]. One of the most interesting events in $\mathrm{AD}$ is that mitochondrial oxidative stress occurs early in $\mathrm{AD}$ progression, before the onset of amyloidbeta pathology $[27,28]$. Oxidative stress was also reported in the mitochondria of other tissues different to the brain such as platelets and fibroblasts from $\mathrm{AD}$ patients $[29,30]$.

Free radicals (compounds with an unpaired electron) or ROS are a normal part of metabolism. Mitochondria are the major source of ROS, and, in fact, mitochondrial dysfunction as well as hypometabolism has long been implicated in the onset of the familial and sporadic forms of AD [31]. mtDNA defects have also been linked to an increased incidence of $\mathrm{AD}$ [32]. Quantitative morphometric, molecular, and cellular analysis of mitochondria shows increased abnormal and damaged mitochondria in $\mathrm{AD}[33,34]$. Energy deficiency and mitochondrial dysfunction have been recognized as a prominent, early event in $\mathrm{AD}$.
Mitochondrial abnormalities have been found both in neurons and astrocytes [35-37], suggesting that both neurons and astrocytes might be damaged by free radicals in the AD brain. Superoxide radicals might be produced in mitochondrial electron transport chain complexes I and III [38] and in components of the Krebs cycle, including aketoglutarate dehydrogenase [39]. In addition, superoxide radicals might be generated in the outer mitochondrial membrane. $\mathrm{H}_{2} \mathrm{O}_{2}$ and superoxide radicals, released from the mitochondrial matrix and from the inner and outer mitochondrial membranes, might be carried to the cytoplasm and, ultimately, might lead to the oxidation of cytoplasmic proteins [26].

Several lines of evidence suggest that APP and amyloidbeta are factors contributing to mitochondrial dysfunction in $\mathrm{AD}$ (Figure 2). Mitochondria were found to be the target both for amyloid precursor protein (APP) that accumulates in the mitochondrial import channels and for amyloid-beta that interacts with several proteins inside mitochondria and leads to mitochondrial dysfunction [30]. Multiple lines of evidence support APP and amyloid-beta as contributing factors to mitochondrial dysfunction in AD: both APP and amyloid-beta are present in mitochondrial membrane and interact with mitochondrial proteins, block mitochondrial import channels, impair mitochondrial transport, disrupt the electron transfer chain, increase ROS levels, and cause mitochondrial damage.

With regard to localization of APP in mitochondria, it was demonstrated that APP formed stable $480 \mathrm{kDa}$ complexes with the translocase of the outer mitochondrial membrane 40 (TOM40) import channel and a supercomplex of approximately $620 \mathrm{kDa}$ with both mitochondrial TOM 40 and the translocase of the inner mitochondrial membrane 23 (TIM23) import channel TIM23 in an N (in mitochondria) -C (out of cytoplasm) orientation [40]. Interestingly, in brain tissues of AD-affected subjects, APP localized with mitochondria fraction, associated to TOM40 and TIM23, in a translocation-arrested manner, that may prevent import of de novo synthesised nuclear-encoded mitochondrial protein, such as subunits of the electron transport chain [27].

In agreement with the intracellular localization of APP, cell studies showed mitochondrial accumulation of amyloidbeta in AD patients and APP mouse transgenic mouse brain [28]. In transgenic APP mice, mitochondrial amyloid-beta accumulation increased at around 4 months of age, well before the formation of plaques [41]. In total, these findings are further in line with the recently proposed hypothesis of an intracellular amyloid-beta toxicity cascade which suggests that the toxic amyloid-beta species intervening in molecular and biochemical abnormalities may be intracellular soluble aggregates instead of extracellular, insoluble plaques [42].

APP and amyloid-beta may block mitochondrial translocation of nuclear-encoded proteins [32], such as components of the electron transport chain [43-45], impairing mitochondrial function. Intramitochondrial amyloid-beta is able to perturb mitochondrial function in several ways by directly influencing extracellular transport chain complex activities [46], impairing mitochondrial dynamics [11], or disturbing calcium storage $[47,48]$, thus increasing apoptotic pathways 


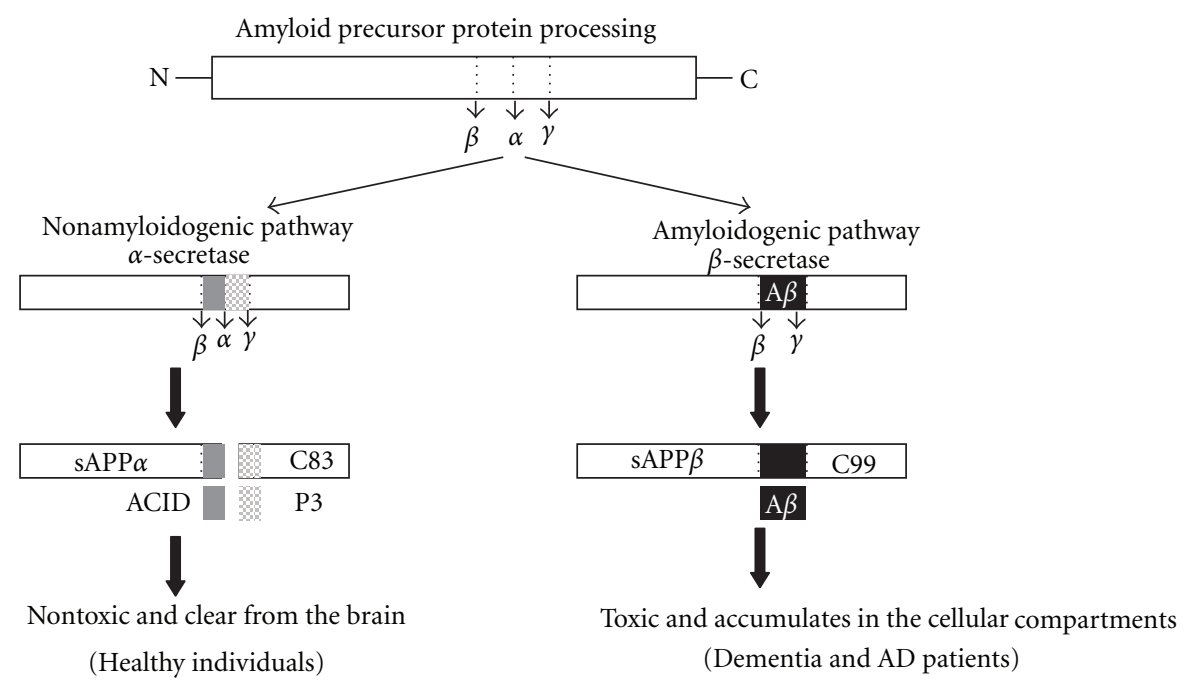

Figure 2: APP processing in nondemented healthy individuals and AD patients. APP processing occurs by two pathways: a betasecretase-based amyloidogenic and alpha-secretase-based nonamyloidogenic pathway. In the nonamyloidogenic pathway (left), cleavage occurs by alpha-secretase within the amyloid-beta domain and generates the large soluble N-terminal fragment (sAPP $\alpha$ ) and a nonamyloidogenic C-terminal fragment of 83 amino acid residues (C83). Further cleavage of this C-terminal fragment by $\gamma$-secretase generates the nonamyloidogenic peptide (P3) and APP intracellular domain (ACID). These products are nontoxic. The non-amyloidogenic $\alpha$-secretase pathway occurs in over $90 \%$ of humans, and these individuals generally do not develop dementia. In the amyloidogenic pathway (right), cleavage occurs by $\beta$-secretase at the beginning of the amyloid-beta domain and generates a soluble N-terminus fragment (sAPP $\beta$ ) and amyloidogenic C-terminal fragment of 99 residues (C99). This C-terminal fragment is further cleaved by $\gamma$-secretase and generates amyloidbeta. The amyloidogenic pathway occurs in approximately $10 \%$ of total humans, and these individuals might develop dementia and AD.

[49]. Moreover, amyloid-beta interacts with mitochondrial matrix components inducing an improper mitochondrial complex function leads to a decreased mitochondrial membrane potential of the organelle [50] and impairing ATP formation [51].

In APP processing, monomeric amyloid-beta forms oligomers in synaptic terminals. Oligomeric amyloid-beta is hypothesized to enter in the mitochondria by penetrating the membrane because amyloid-beta is enriched at synaptic terminals [52]. In support of the hypothesis that APP and amyloid-beta enter mitochondria, several studies have found APP and its derivatives (monomeric and oligomeric forms of amyloid-beta) in mitochondrial membranes [53, 54]. Amyloid-beta normally interact with the mitochondrial matrix protein, amyloid-beta-binding alcohol dehydrogenase (ABAD), leading to mitochondrial dysfunction [9]. Caspersen et al. [41] showed amyloid-beta in mitochondria from postmortem brain specimens of $\mathrm{AD}$ patients and an accumulation of amyloid-beta in the brain mitochondria from APP mice. With digitonin fractionation analysis of isolated mitochondria from APP mice revealed amyloid-beta in outer and inner mitochondrial membranes and matrix and that mitochondrial amyloid-beta decreases cytochrome oxidase activity and increases free radical production and carbonyl proteins [28].

Further, in the amyloid interactions in mitochondrial proteins, two more studies found that APP interacts directly with mitochondrial proteins. It was demonstrated that mitochondrial ATP synthase subunit is a binding partner of the extracellular domain of APP and amyloid-beta [50]. Transfection of APP-deficient neuroblastoma cells with APP resulted in increased surface localization of the ATP synthase a-subunit and in extracellular APP and amyloid-beta inhibiting the extracellular generation of ATP. In another study, the authors found that nonglycosylated full-length and C-terminal-truncated APP accumulates in the protein import channels of mitochondria of human AD brains but not in age-matched controls [27]. The accumulation of APP across mitochondrial import channels inhibited the entry of nuclear-encoded cytochrome c-oxidase subunits IV and $\mathrm{Vb}$ proteins and was associated with decreased cytochrome oxidase and increased free radical production.

\section{Mitochondrial DNA (mtDNA) Changes in AD}

It is well documented that mtDNA changes are responsible for aging phenotypes [55-57]. For example, many tissues from aged individuals have a lower respiratory function compared with those from younger individuals. It has been hypothesized that ongoing oxidative damage to mtDNA may be the underlying mechanism for cellular senescence [58]. Since mtDNA repair mechanisms are limited and because mtDNA is situated in close proximity to the site of ROS production, mtDNA is more vulnerable to oxidative damage than nuclear DNA [59]. With age, oxidation of mtDNA increases compared to nuclear DNA leading to an agedependent accumulation of mtDNA mutations [60].

Point mutations and deletions in mtDNA are highly prevalent in aged cells, and there is evidence that 8-hydroxy2 - deoxyguanosine (damaged DNA) is more prevalent in 
aged tissues [55]. Further, mice carrying an mtDNA mutation (in the DNA polymerase-g gene) showed features of aging and reduced lifespan, suggesting that mtDNA changes are crucial for aging phenotypes [56].

Defects in mtDNA have not only been found in elderly persons without $\mathrm{AD}$ but also in $\mathrm{AD}$ patients $[61,62]$ and have been associated with decreased cytochrome oxidase activity in non- $\mathrm{AD}$ aging and aging $\mathrm{AD}$ brains. One recent study found that somatic mtDNA control region mutations are elevated in $\mathrm{AD}$ patients [63]. These mutations would lead to an overall reduction in mtDNA copy number which would result in a decrease in oxidative phosphorylation. In addition, a mutation that affects L-strand transcription was also discovered. This mutation inhibits complex I respiration which leads to increased ROS production, decreased membrane potential, and subsequent calcium deregulation. The effects of these mutations may lead to opening of the mitochondrial permeability transition pore and subsequent neuronal death.

Increased ROS levels act at multiple levels to impair mitochondrial function: they induce mtDNA mutations [64] that consequently negatively influence mitochondrial function [65], enhance amyloid-beta production by guiding APP cleavage pathway toward the amyloidogenesis [66], increase lipid peroxidation [67, 68], activate mitophagy, leading to a reduced mitochondrial number [36], and augment tau hyperphosphorylation and NFT formation impairing organelle trafficking and neuronal function finally leading to apoptosis.

Using quantitative RT-PCR techniques, it was measured mRNA expression of 11 mitochondrial-encoded genes in patients with early $\mathrm{AD}$ and with definite $\mathrm{AD}$, as well as in age-matched control subjects. This interesting analysis revealed a downregulation of mitochondrial genes in complex I of OXPHOS (oxidative phosphorylation) in brain of $\mathrm{AD}$ patients. Complex I showed a down regulation of mitochondrial genes, whereas complexes III and IV showed increased mRNA expressions in these $\mathrm{AD}$ brains, suggesting a great demand on energy production [35]. In a previous paragraph, we reported a decrease of cytochrome oxidase in the mitochondria from platelets, fibroblasts, and brains of $\mathrm{AD}$ patients. To compensate for the loss of cytochrome oxidase, mitochondrial-encoded genes might be activated in the surviving brain neurons of $\mathrm{AD}$, for those patients this chain of events has been interpreted as a compensatory response [26].

\section{Mitochondria and Synaptic Damage in AD}

Synaptic degeneration is an early pathological feature in $\mathrm{AD}$ and is closely correlated to impaired cognitive function and memory loss. Recent studies suggest that involvement of amyloid-beta peptide in synaptic mitochondrial alteration underlies these synaptic lesions. Based on recent findings in human $\mathrm{AD}$ subjects, $\mathrm{AD}$ animal models, and $\mathrm{AD}$ cellular models, synaptic mitochondria undergo multiple malfunctions including amyloid-beta accumulation, increased oxidative stress, decreased respiration, and compromised calcium handling capacity, all of which occur earlier than changes seen in nonsynaptic mitochondria prior to predominant $\mathrm{AD}$ pathology. Of note, the impact of amyloid-beta on mitochondrial motility and dynamics exacerbates synaptic mitochondrial alterations.

Mitochondrial number is indeed very high in neurons, and mitochondria are especially enriched in synapses. Due to the limited glycolytic capacity of neurons, these cells are highly dependent on mitochondria function for energy production. Thus, the importance of synaptic mitochondria in supporting synapses and the high vulnerability of synaptic mitochondria to amyloid-beta make them a promising target of new therapeutic strategy for AD.

Synaptic mitochondria are synthesized in neuronal soma; they are then transported to dendrites and axones, are distributed abundantly around synapses where mitochondria modulate calcium balance, and actively provide energy to fuel the synaptic function $[69,70]$. If mitochondria localized in the cell body are damaged or are otherwise degraded, such as by aging or by amyloid-beta, these defective mitochondria might be transported to synaptic terminals by natural mitochondrial trafficking, where they produce low levels of ATP owing to their degradation. Synaptic mitochondria thus undergo constant activation to maintain synaptic function. Defects in synaptic mitochondria obviously compromise synaptic function being very vulnerable to accumulative damages.

Mitochondrial dysfunction and synaptic damage are early pathological features of AD. Gillardon et al. found amyloid-beta oligomers in synaptosomal mitochondrial fractions and decreased energy metabolism in $\mathrm{AD}$ transgenic mice [71]. Abnormalities of mitochondrial function, including decreased mitochondrial respiration, ROS generation, and hypometabolism, occur in the $\mathrm{AD}$ brain $[39,72]$ and in brains of $\mathrm{AD}$ mouse models [73, 74]. Amyloid-beta accumulation in the synapses directly disturbs mitochondrial function, causing oxidative stress, decreased ATP, and increased $\mathrm{Ca}^{2+}$ influx $[9,75]$. Furthermore, the interaction of mitochondrial amyloid-beta with its binding proteins, such as ABAD and CypD $[9,75]$, exacerbates amyloid-betainduced mitochondria and neuronal stress and malfunction.

Further, it was recently showed that amyloid-beta impaired synaptic mitochondrial distribution, axonal mitochondrial mobility, and increased axonal mitochondrial fragmentation. Interestingly, anterograde movements were more susceptible to amyloid-beta insult. These promising findings are in agreement with those of recent studies indicating that amyloid-beta causes rapid and severe impairment of mitochondrial transport [47] and alters mitochondrial dynamics [76].

Synaptic terminals are sites of high energy demand and require high levels of cellular ATP for neurotransmitter exocytosis and the potentiation of neurotransmitter release. These studies suggest that in an amyloid-betarich environment, overt mitochondrial dysfunction occurs and that mitochondria provide a direct site for amyloidbeta-mediated cellular perturbation, causing synaptic mitochondrial dysfunction in $\mathrm{AD}$. However, it is not yet known whether amyloid-beta accumulates predominantly in synaptic mitochondria and whether synaptic mitochondria 
enriched for amyloid-beta are more vulnerable, although it is well known that the increase in oxidative damage exhibited by synaptic mitochondria might affect neurotransmission and synaptic damage and loss and might be ultimately responsible for cognitive decline in $\mathrm{AD}$ patients.

In view of the critical role of synaptic mitochondria in energy production, synaptic calcium buffering, and regulation of synaptic function, impaired movement to and sequestration of mitochondria at synapses by amyloid-beta could be a mechanism of the pathogenesis at the distal synapses, together with early changes in synaptic mitochondrial function.

\section{Mitochondrial Dysfunction in Choroid Plexus}

In $\mathrm{AD}$ is very well documented the intracellular deposits of amyloid-beta in the brain parenchyma; however, amyloidbeta also accumulates in choroid plexus epithelial cells [77] and in cerebrovascular walls, where it induces blood-brain barrier disruption [78]. Several studies have shown that amyloid-beta alters transmembrane and cytoplasmic tight junction proteins in brain microvessel endothelial cells and choroid plexus, which ultimately leads to disruption in the integrity of the blood-brain barrier $[79,80]$.

Abnormal patterns of mitochondrial stress protein expression are found in the choroid plexus and BBB of $\mathrm{AD}$ subjects. There was a trend towards increased expression of HSP60, a mitochondrial stress protein, compatible with mitochondrial pathology recently documented in choroid plexus of $\mathrm{AD}$ [81]. It was recently described in choroid plexus homogenates from 27 cases with AD-related pathology in different stages (stages I/0, III-IV/0-B and V-VI/B-C) increased carboxyethyl-lysine (CEL) and carboxymethyllysine (CML) expression in $\mathrm{AD}$ cases stages IVB and $\mathrm{V}$ VI/B-C when compared to controls and cases with ADrelated pathology. Interestingly, the authors suggest that other factors in addition to local fibrillar amyloid-beta were associated with oxidative damage in the choroid plexus. Though two-dimensional gel electrophoresis in combination with mass spectrometry identified other proteins as targets of increased oxidative damage in $\mathrm{AD}$ (tyrosine 3/tryptophan 5-monooxygenase activation protein, zeta polypeptide, tropomyosin 3 isoform, and apolipoprotein A-II) [82].

Recent results from our laboratory have suggested direct relationship between amyloid-beta accumulation at the choroid plexus epithelium and the development of functional and structural dysfunctions $[77,83]$. In addition, we demonstrated the existence of a link between amyloidbeta-induced choroid plexus cell death, increased production of nitric oxide (NO), and mitochondrial dysfunction in the choroid plexus of patients with AD and APP/PS1 mice [83]. In $\mathrm{AD}$ patients and $\mathrm{APP} / \mathrm{Ps}$ mice, we found an alteration induced by amyloid-beta of the enzyme activity of the respiratory chain complex IV in the choroid plexus epithelial cells [84]. Accumulation of amyloid-beta peptides is a critical event in the pathology of $\mathrm{AD}$, because they induce multiple neurotoxic effects, including mitochondrial dysfunction.
Based on these results, we considered a reduction of amyloidbeta with gelsolin such as primary therapeutic target [85].

Gelsolin is an amyloid-beta binding protein that inhibits apoptosis, although the underlying mechanism is unclear. We observed that gelsolin reduces brain amyloid-beta burden in the APP/Ps1 mice, accompanied by an inhibition of nitricoxide production and cell death, not only in the choroid plexus but also in the cerebral cortex. Gelsolin levels restored the impaired mitochondrial activity, resulting in the increase of cytochrome c-oxidase activity [84].

Additionally, these result demonstrated that gelsolin plays an important role in decreasing amyloid-beta-induced cytotoxicity by inhibiting nitric oxide production and apoptotic mitochondrial changes. All these promising findings make gelsolin an appealing tool for the prophylactic treatment of AD.

\section{Conclusions}

Based on findings from in vitro and in vivo studies, it has been proposed that amyloid-beta has a significant role in synaptic dysfunction and cognitive decline in AD patients. Among other facts, amyloid-beta accumulates at synaptic terminals and enters the mitochondria, especially in the synaptic mitochondria. Mitochondria have been implicated in the neurodegenerative diseases and the onset and progression of age-associated diseases since decades. Mitochondria are the major source of energy for the brain [86]. The accumulation of mtDNA changes might increase ROS production and reduce mitochondrial ATP in an age-dependent manner. Recent studies of neurons from postmortem $\mathrm{AD}$ brain specimens and from transgenic AD mouse brain specimens suggest that oxidative damage induces amyloid-beta production.

Studies of postmortem brains from $\mathrm{AD}$ patients and transgenic mouse models of $\mathrm{AD}$ suggest that oxidative damage, induced by amyloid-beta, is associated with mitochondria early in AD progression. Amyloid-beta present within mitochondria may provide a direct link between mitochondrial dysfunction in $\mathrm{AD}$ and pathogenic amyloidbeta. Amyloid-beta associated with mitochondria may be deposited at several locations, and yet nobody has known if amyloid-beta enters only in presynaptic mitochondria, postsynaptic mitochondria, or both. Interestingly, amyloidbeta is not present exclusively on the outer mitochondrial membrane and also might be present at that site to influence the interaction of multiple cytosolic proteins (including those of the bcl2 family) with mitochondria, as well as affecting the receptor binding of cargo targeted for import into the organelle. Amyloid-beta and APP are reported to localize to mitochondrial membranes, block the transport of nuclear-encoded mitochondrial proteins to mitochondria, interact with mitochondrial proteins, disrupt electron transport chain activities, increase ROS production, cause mitochondrial damage, and prevent neurons from functioning normally.

The mechanisms of amyloid-beta and APP transport into mitochondrial membranes are unclear. Amyloid-beta 
accumulates at synaptic terminals and impairs synaptic function and also enters synaptic mitochondria and causes damage. Mitochondrial damage is expected to be greater in synaptic mitochondria than in cell body mitochondria. The damaged, synaptic mitochondria might not satisfy the high energy demands required at synapses, which might lead to impaired neurotransmission and, ultimately, to cognitive failure. Based on these concepts, therapies targeting basic mitochondrial processes, such as energy metabolism or free radical generation, or specific interactions of diseaserelated proteins with mitochondria, hold great promise and future. In $\mathrm{AD}$, tremendous progress has been made in developing therapeutic strategies to decrease amyloid-beta production and toxicity. However, further research is needed to develop molecules that target intact amyloid-beta in brain neurons affected by AD. Further research is also needed to test the efficacies of mitochondrially targeted antioxidants in $\mathrm{AD}$ mouse models. Future experiments that focus on the functional association of mitochondria with APP and amyloid-beta might be useful for identifying mitochondrial drug targets.

\section{Conflict of Interests}

None of the authors of this paper have any financial interest that has influenced the results or interpretation of this paper.

\section{Acknowledgments}

The authors thank Tania Vazquez for editorial assistance. This work was supported by Grants from Xunta de Galicia (INCITE2009, 09CSA051905PR), Spanish Institute of Health Carlos III (PI-11/00842 and PI10/02628), and "Isidro Parga Pondal" programme. The authors also apologize to those authors whose original work could not be cited due to space limitations.

\section{References}

[1] OECD, "Dementia prevalence," in OECD, Health at a Glance: Europe 2010, pp. 54-55, OECD, 2010.

[2] L. Bertram and R. E. Tanzi, "The genetic epidemiology of neurodegenerative disease," The Journal of Clinical Investigation, vol. 115, no. 6, pp. 1449-1457, 2005.

[3] C. P. Ferri, M. Prince, C. Brayne et al., "Global prevalence of dementia: a Delphi consensus study," The Lancet, vol. 366, no. 9503, pp. 2112-2117, 2005.

[4] American Health Assistance Foundation. Alzheimer disease research: about Alzheimer, http://www.ahaf.org/alzheimers/.

[5] O. V. Forlenza, B. S. Diniz, and W. F. Gattaz, "Diagnosis and biomarkers of predementia in Alzheimer's disease," BMC Medicine, vol. 8, article 89, 2010.

[6] J. Gotz, A. Eckert, M. Matamales et al., "Modes of Ab toxicity in Alzheimer's disease," Cellular and Molecular Life Science, vol. 68, no. 20, pp. 3359-3375, 2011.

[7] A. Eckert, S. Hauptmann, I. Scherping et al., "Oligomeric and fibrillar species of $\beta$-amyloid (A $\beta 42)$ both impair mitochondrial function in P301L tau transgenic mice," Journal of Molecular Medicine, vol. 86, no. 11, pp. 1255-1267, 2008.
[8] S. Hauptmann, I. Scherping, S. Dröse et al., "Mitochondrial dysfunction: an early event in Alzheimer pathology accumulates with age in AD transgenic mice," Neurobiology of Aging, vol. 30, no. 10, pp. 1574-1586, 2009.

[9] J. W. Lustbader, M. Cirilli, C. Lin et al., "ABAD directly links $\mathrm{A} \beta$ to mitochondrial toxicity in Alzheimer's disease," Science, vol. 304, no. 5669, pp. 448-452, 2004.

[10] T. A. Clark, H. P. Lee, R. K. Rolston et al., "Oxidative stress and its implications for future treatments and management of alzheimer disease," International Journal of Biomedical Science, vol. 6, no. 3, pp. 225-227, 2010.

[11] X. Wang, B. Su, S. L. Siedlak et al., "Amyloid- $\beta$ overproduction causes abnormal mitochondrial dynamics via differential modulation of mitochondrial fission/fusion proteins," Proceedings of the National Academy of Sciences of the United States of America, vol. 105, no. 49, pp. 19318-19323, 2008.

[12] N. Bogdanovic, M. Zilmer, K. Zilmer, A. Rehema, and E. Karelson, "The Swedish APP670/671 Alzheimer's disease mutation: the first evidence for strikingly increased oxidative injury in the temporal inferior cortex," Dementia and Geriatric Cognitive Disorders, vol. 12, no. 6, pp. 364-370, 2001.

[13] D. Praticò, K. Uryu, S. Leight, J. Q. Trojanoswki, and V. M. Y. Lee, "Increased lipid peroxidation precedes amyloid plaque formation in an animal model of alzheimer amyloidosis," Journal of Neuroscience, vol. 21, no. 12, pp. 4183-4187, 2001.

[14] A. Martínez-García, I. Sastre, M. Recuero et al., "PLA2G3, a gene involved in oxidative stress induced death, is associated with Alzheimer's disease," Journal of Alzheimer's Disease, vol. 22, no. 4, pp. 1181-1187, 2010.

[15] T. Vargas, C. Ugalde, C. Spuch et al., "A $\beta$ accumulation in choroid plexus is associated with mitochondrial-induced apoptosis," Neurobiology of Aging, vol. 31, no. 9, pp. 15691581, 2010.

[16] E. Perez-Gracia, R. Blanco, M. Carmona, E. Carro, and I. Ferrer, "Oxidative stress damage and oxidative stress responses in the choroid plexus in Alzheimer's disease," Acta Neuropathologica, vol. 118, no. 4, pp. 497-504, 2009.

[17] T. G. Freya and C. A. Mannellab, "The internal structure of mitochondria," Trends in Biochemical Sciences, vol. 25, no. 7, pp. 319-324, 2000.

[18] S. Anderson, A. T. Bankier, and B. G. Barrell, "Sequence and organization of the human mitochondrial genome," Nature, vol. 290, no. 5806, pp. 457-465, 1981.

[19] R. E. Giles, H. Blanc, H. M. Cann, and D. C. Wallace, "Maternal inheritance of human mitochondrial DNA," Proceedings of the National Academy of Sciences of the United States of America, vol. 77, no. 11, pp. 6715-6719, 1980.

[20] X. J. Chen and R. A. Butow, "The organization and inheritance of the mitochondrial genome," Nature Reviews Genetics, vol. 6, no. 11, pp. 815-825, 2005.

[21] J. A. Cam, C. V. Zerbinatti, J. M. Knisely, S. Hecimovic, Y. $\mathrm{Li}$, and $\mathrm{G}$. $\mathrm{Bu}$, "The low density lipoprotein receptorrelated protein $1 \mathrm{~B}$ retains $\beta$-amyloid precursor protein at the cell surface and reduces amyloid- $\beta$ peptide production," The Journal of Biological Chemistry, vol. 279, no. 28, pp. 2963929646, 2004.

[22] Q. Liu, C. V. Zerbinatti, J. Zhang et al., "Amyloid precursor protein regulates brain apolipoprotein $\mathrm{E}$ and cholesterol metabolism through lipoprotein receptor LRP1," Neuron, vol. 56 , no. 1 , pp. 66-78, 2007.

[23] E. Waldron, C. Heilig, A. Schweitzer et al., "LRP1 modulates APP trafficking along early compartments of the secretory pathway," Neurobiology of Disease, vol. 31, no. 2, pp. 188-197, 2008. 
[24] X. Alvira-Botero, R. Perez-Gonzalez, C. Spuch et al., "Megalin interact with APP and the intracellular adaptor protein FE65 in neurons," Molecular and Cellular Neuroscience, vol. 45, pp. 306-315, 2010.

[25] R. Deane, S. D. Yan, R. K. Submamaryan et al., "RAGE mediates amyloid- $\beta$ peptide transport across the blood-brain barrier and accumulation in brain," Nature Medicine, vol. 9, no. 7, pp. 907-913, 2003.

[26] P. H. Reddy, "Amyloid precursor protein-mediated free radicals and oxidative damage: implications for the development and progression of Alzheimer's disease," Journal of Neurochemistry, vol. 96, no. 1, pp. 1-13, 2006.

[27] L. Devi, B. M. Prabhu, D. F. Galati, N. G. Avadhani, and H. K. Anandatheerthavarada, "Accumulation of amyloid precursor protein in the mitochondrial import channels of human Alzheimer's disease brain is associated with mitochondrial dysfunction," Journal of Neuroscience, vol. 26, no. 35, pp. 90579068, 2006.

[28] M. Manczak, T. S. Anekonda, E. Henson, B. S. Park, J. Quinn, and P. H. Reddy, "Mitochondria are a direct site of $\mathrm{A} \beta$ accumulation in Alzheimer's disease neurons: implications for free radical generation and oxidative damage in disease progression," Human Molecular Genetics, vol. 15, no. 9, pp. 1437-1449, 2006.

[29] M. F. Beal, "Mitochondria take center stage in aging and neurodegeneration," Annals of Neurology, vol. 58, no. 4, pp. 495-505, 2005.

[30] P. H. Reddy, "Mitochondrial dysfunction in aging and Alzheimer's disease: strategies to protect neurons," Antioxidants and Redox Signaling, vol. 9, no. 10, pp. 1647-1658, 2007.

[31] K. Nuramaki, N. Murata, Y. Noda et al., "SOD1 deficiency drives amyloid beta oligomerization and memory loss in a mouse model of Alzheimer's diserase," The The Journal of Biological Chemistry. In press.

[32] M. Mancuso, D. Orsucci, A. LoGerfo, V. Calsolaro, and G. Siciliano, "Clinical features and pathogenesis of Alzheimer's disease: involvement of mitochondria and mitochondrial DNA," Advances in Experimental Medicine and Biology, vol. 685, pp. 34-44, 2010.

[33] D. F. F. Silva, A. R. Esteves, D. M. Arduino, C. R. Oliveira, and S. M. Cardoso, "Amyloid- $\beta$-induced mitochondrial dysfunction impairs the autophagic lysosomal pathway in a tubulin dependent pathway," Journal of Alzheimer's Disease, vol. 26, no. 3, pp. 565-581, 2011.

[34] A. Eckert, K. Schmitt, and J. Gotz, "Mitochondrial dysfunction, the beginning of the end in Alzheimer's disease? Separate and synergistic modes of tau and amyloid beta toxicity," Alzheimer's Research \& Therapy, vol. 3, article 15, 2011.

[35] M. Manczak, B. S. Park, Y. Jung, and P. H. Reddy, "Differential expression of oxide phosphorylation genes in patients with Alzheimer's disease: implications for early mitochondrial dysfunction and oxidative damage," NeuroMolecular Medicine, vol. 5, no. 2, pp. 147-162, 2004.

[36] K. Hirai, G. Aliev, A. Nunomura et al., "Mitochondrial abnormalities in Alzheimer's disease," Journal of Neuroscience, vol. 21, no. 9, pp. 3017-3023, 2001.

[37] A. Y. Abramov, L. Canevari, and M. R. Duchen, "betaamyloid peptides induce mitochondrial dysfunction and oxidative stress in astrocytes and death of neurons," Journal of Neuroscience, vol. 24, no. 2, pp. 565-575, 2004.

[38] G. E. Gibson and Q. Shi, "A mitocentric view of Alzheimer's disease suggests multi-faceted treatments," Journal of Alzheimer's Disease, vol. 20, no. 2, pp. S591-S607, 2010.
[39] M. T. Lin and M. F. Beal, "Mitochondrial dysfunction and oxidative stress in neurodegenerative diseases," Nature, vol. 443, no. 7113, pp. 787-795, 2006.

[40] H. K. Anandatheerthavarada, G. Biswas, M. A. Robin, and N. G. Avadhani, "Mitochondrial targeting and a novel transmembrane arrest of Alzheimer's amyloid precursor protein impairs mitochondrial function in neuronal cells," Journal of Cell Biology, vol. 161, no. 1, pp. 41-54, 2003.

[41] C. Caspersen, N. Wang, J. Yao et al., "Mitochondrial A $\beta$ : a potential focal point for neuronal metabolic dysfunction in Alzheimer's disease," The FASEB Journal, vol. 19, no. 14, pp. 2040-2041, 2005.

[42] P. Fernández-Vizarra, A. P. Fernández, S. Castro-Blanco et al., "Intra- and extracellular A $\beta$ and PHF in clinically evaluated cases of Alzheimer's disease," Histology and Histopathology, vol. 19, no. 3, pp. 823-844, 2004.

[43] W. D. Parker, C. M. Filley, and J. K. Parks, "Cytochrome oxidase deficiency in Alzheimer's disease," Neurology, vol. 40, no. 8, pp. 1302-1303, 1990.

[44] W. D. Parker and J. K. Parks, "Cytochrome c oxidase in Alzheimer's disease brain: purification and characterization," Neurology, vol. 45, no. 3, pp. 482-486, 1995.

[45] S. M. Cardoso, I. Santana, R. H. Swerdlow, and C. R. Oliveira, "Mitochondria dysfunction of Alzheimer's disease cybrids enhances A $\beta$ toxicity," Journal of Neurochemistry, vol. 89, no. 6, pp. 1417-1426, 2004.

[46] V. Rhein, X. Song, A. Wiesner et al., "Amyloid- $\beta$ and tau synergistically impair the oxidative phosphorylation system in triple transgenic Alzheimer's disease mice," Proceedings of the National Academy of Sciences of the United States of America, vol. 106, no. 47, pp. 20057-20062, 2009.

[47] R. J. Mark, Z. Pang, J. W. Geddes, K. Uchida, and M. P. Mattson, "Amyloid $\beta$-peptide impairs glucose transport in hippocampal and cortical neurons: involvement of membrane lipid peroxidation," Journal of Neuroscience, vol. 17, no. 3, pp. 1046-1054, 1997.

[48] M. P. Mattson, "Pathways towards and away from Alzheimer's disease," Nature, vol. 430, no. 7000, pp. 631-639, 2004.

[49] H. Du, L. Guo, W. Zhang, M. Rydzewska, and S. Yan, "Cyclophilin D deficiency improves mitochondrial function and learning/memory in aging Alzheimer disease mouse model," Neurobiology of Aging, vol. 32, pp. 398-406, 2009.

[50] U. Keil, S. Hauptmann, A. Bonert, I. Scherping, A. Eckert, and W. E. Müller, "Mitochondrial dysfunction induced by disease relevant $\mathrm{A} \beta \mathrm{PP}$ and tau protein mutations," Journal of Alzheimer's Disease, vol. 9, no. 2, pp. 139-146, 2006.

[51] P. I. Moreira, C. Carvalho, X. Zhu, M. A. Smith, and G. Perry, "Mitochondrial dysfunction is a trigger of Alzheimer's disease pathophysiology," Biochimica et Biophysica Acta, vol. 1802, no. 1, pp. 2-10, 2010.

[52] C. G. Glabe and R. Kayed, "Common structure and toxic function of amyloid oligomers implies a common mechanism of pathogenesis," Neurology, vol. 66, no. 2, pp. S74-S78, 2006.

[53] S. Chang, T. R. Ma, R. D. Miranda, M. E. Balestra, R. W. Mahley, and Y. Huang, "Lipid- and receptor-binding regions of apolipoprotein E4 fragments act in concert to cause mitochondrial dysfunction and neurotoxicity," Proceedings of the National Academy of Sciences of the United States of America, vol. 102, no. 51, pp. 18694-18699, 2005.

[54] E. A. Schon and E. Area-Gomez, "Is Alzheimer's disease a disorder of mitochondria-associated membranes?" Journal of Alzheimer's Disease, vol. 20, no. 2, pp. S281-S292, 2010. 
[55] G. C. Kujoth and T. A. Prolla, "Evolving insight into the role of mitochondrial DNA mutations in aging," Experimental Gerontology, vol. 43, no. 1, pp. 20-23, 2008.

[56] G. C. Kujoth, P. C. Bradshaw, S. Haroon, and T. A. Prolla, "The role of mitochondrial DNA mutations in mammalian aging," PLoS Genetics, vol. 3, no. 2, article e24, 2007.

[57] S. A. Dogan and A. Trifunovic, "Modelling mitochondrial dysfunction in mice," Physiological Research, vol. 60, pp. 6170, 2011.

[58] I. Bratic and A. Trifunovic, "Mitochondrial energy metabolism and ageing," Biochimica et Biophysica Acta, vol. 1797, no. 6-7, pp. 961-967, 2010.

[59] S. Z. Imam, B. Karahalil, B. A. Hogue, N. C. Souza-Pinto, and V. A. Bohr, "Mitochondrial and nuclear DNA-repair capacity of various brain regions in mouse is altered in an agedependent manner," Neurobiology of Aging, vol. 27, no. 8, pp. 1129-1136, 2006.

[60] J. Sastre, A. Millan, J. G. De La Asuncion et al., "A ginkgo biloba extract (EGb 761) prevents mitochondrial aging by protecting against oxidative stress," Free Radical Biology and Medicine, vol. 24, no. 2, pp. 298-304, 1998.

[61] M. T. Lin, D. K. Simon, C. H. Ahn, L. M. Kim, and M. Flint Beal, "High aggregate burden of somatic mtDNA point mutations in aging and Alzheimer's disease brain," Human Molecular Genetics, vol. 11, no. 2, pp. 133-145, 2002.

[62] P. E. Coskun, M. F. Beal, and D. C. Wallace, "Alzheimer's brains harbor somatic mtDNA control-region mutations that suppress mitochondrial transcription and replication," Proceedings of the National Academy of Sciences of the United States of America, vol. 101, no. 29, pp. 10726-10731, 2004.

[63] P. E. Coskun, J. Wyrembak, O. Derbereva et al., "Systemic mitochondrial dysfunction and the etiology of Alzheimer's disease and down syndrome dementia," Journal of Alzheimer's Disease, vol. 20, no. 2, pp. S293-S310, 2010.

[64] J. M. Shoffner, M. D. Brown, A. Torroni et al., "Mitochondrial DNA variants observed in Alzheimer disease and Parkinson disease patients," Genomics, vol. 17, no. 1, pp. 171-184, 1993.

[65] R. H. Swerdlow, "Mitochondria in cybrids containing mtDNA from persons with mitochondriopathies," Journal of Neuroscience Research, vol. 85, no. 15, pp. 3416-3428, 2007.

[66] M. Newman, I. F. Musgrave, and M. Lardelli, "Alzheimer's disease: amyloidogenesis, the presenilins and animal models," Biochimica et Biophysica Acta, vol. 1772, no. 9, pp. 285-297, 2007.

[67] E. Richartz, S. Noda, K. Schott, A. Günthner, P. Lewczuk, and M. Bartels, "Increased serum levels of CD95 in Alzheimer's disease," Dementia and Geriatric Cognitive Disorders, vol. 13, no. 3, pp. 178-182, 2002.

[68] K. Zarkovic, "4-Hydroxynonenal and neurodegenerative diseases," Molecular Aspects of Medicine, vol. 24, no. 4-5, pp. 293 303, 2003.

[69] E. A. Schon and S. Przedborski, "Mitochondria: the next neurodegeneration," Neuron, vol. 70, pp. 1033-1053, 2011.

[70] K. Brickley and F. A. Stephenson, "Trafficking kinesin protein (TRAK)-mediated transport of mitochondria in axons of hippocampal neurons," The Journal of Biological Chemistry, vol. 286, no. 20, pp. 18079-18092, 2011.

[71] F. Gillardon, W. Rist, L. Kussmaul et al., "Proteomic and functional alterations in brain mitochondria from $\operatorname{Tg} 2576$ mice occur before amyloid plaque deposition," Proteomics, vol. 7, no. 4, pp. 605-616, 2007.

[72] H. Du and S. S. Yan, "Mitochondrial medicine for neurodegenerative diseases," International Journal of Biochemistry and Cell Biology, vol. 42, no. 5, pp. 560-572, 2010.
[73] A. Eckert, S. Hauptmann, I. Scherping et al., "Soluble betaamyloid leads to mitochondrial defects in amyloid precursor protein and tau transgenic mice," Neurodegenerative Diseases, vol. 5, no. 3-4, pp. 157-159, 2008.

[74] J. Yao, R. W. Irwin, L. Zhao, J. Nilsen, R. T. Hamilton, and R. D. Brinton, "Mitochondrial bioenergetic deficit precedes Alzheimer's pathology in female mouse model of Alzheimer's disease," Proceedings of the National Academy of Sciences of the United States of America, vol. 106, no. 34, pp. 14670-14675, 2009.

[75] H. Du, L. Guo, F. Fang et al., "Cyclophilin D deficiency attenuates mitochondrial and neuronal perturbation and ameliorates learning and memory in Alzheimer's disease," Nature Medicine, vol. 14, no. 10, pp. 1097-1105, 2008.

[76] X. Wang, B. Su, H. G. Lee et al., "Impaired balance of mitochondrial fission and fusion in Alzheimer's disease," Journal of Neuroscience, vol. 29, no. 28, pp. 9090-9103, 2009.

[77] M. O. Dietrich, C. Spuch, D. Antequera et al., "Megalin mediates the transport of leptin across the blood-CSF barrier," Neurobiology of Aging, vol. 29, no. 6, pp. 902-912, 2008.

[78] R. Deane and B. V. Zlokovic, "Role of the blood-brain barrier in the pathogenesis of Alzheimer's disease," Current Alzheimer Research, vol. 4, no. 2, pp. 191-197, 2007.

[79] C. Spuch and C. Navarro, "Expression and functions of LRP2 in central nervous system: progress in understanding its regulation and the potential use for treatment of neurodegenerative diseases," Recent Pat Immunology, Endocrine and metabolic Agents in Medicinal Chemistry, vol. 10, pp. 249-254, 2010.

[80] C. Spuch and C. Navarro, "Expression and functions of LRP2 in central nervous system: progress in understanding its regulation and the potential use for treatment of neurodegenerative diseases," Endocrine, Metabolic \& Immune Drug Discovery, vol. 4, pp. 190-205, 2010.

[81] S. G. Anthony, H. M. Schipper, R. Tavares et al., "Stress protein expression in the Alzheimer-diseased choroid plexus," Endocrine, Metabolic \& Immune Drug Discovery, vol. 5, pp. 171-177, 2003.

[82] E. Perez-Gracia, R. Blanco, M. Carmona, E. Carro, and I. Ferrer, "Oxidative stress damage and oxidative stress responses in the choroid plexus in Alzheimer's disease," Acta Neuropathologica, vol. 118, no. 4, pp. 497-504, 2009.

[83] T. Vargas, C. Ugalde, C. Spuch et al., "A $\beta$ accumulation in choroid plexus is associated with mitochondrial-induced apoptosis," Neurobiology of Aging, vol. 31, no. 9, pp. 1569$1581,2010$.

[84] T. Vargas, D. Antequera, C. Ugalde, and C. Spuch, "Gelsolin restores $\mathrm{A} \beta$-Induced alterations in choroid plexus epithelium," Journal of Biomedicine and Biotechnology, vol. 2010, Article ID 805405, 7 pages, 2010.

[85] D. Antequera, T. Vargas, C. Ugalde et al., "Cytoplasmic gelsolin increases mitochondrial activity and reduces $\mathrm{A} \beta$ burden in a mouse model of Alzheimer's disease," Neurobiology of Disease, vol. 36, no. 1, pp. 42-50, 2009.

[86] P. H. Reddy and M. F. Beal, "Amyloid beta, mitochondrial dysfunction and synaptic damage: implications for cognitive decline in aging and Alzheimer's disease," Trends in Molecular Medicine, vol. 14, no. 2, pp. 45-53, 2008. 


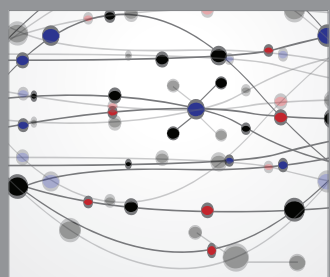

The Scientific World Journal
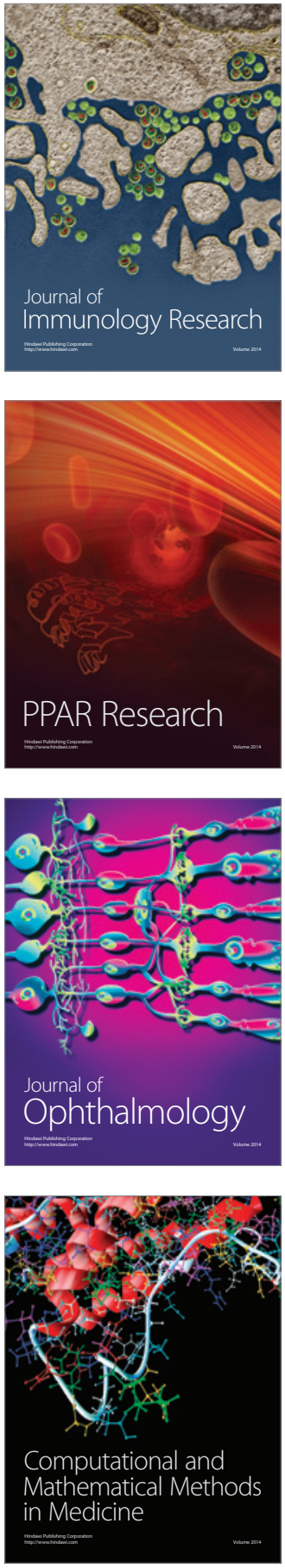

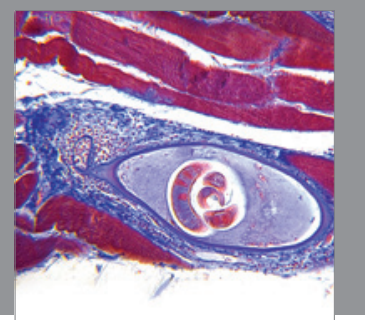

Gastroenterology

Research and Practice
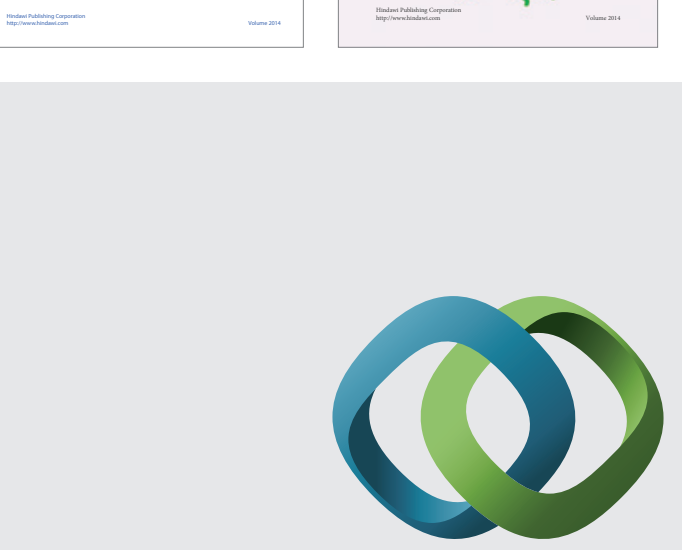

\section{Hindawi}

Submit your manuscripts at

http://www.hindawi.com
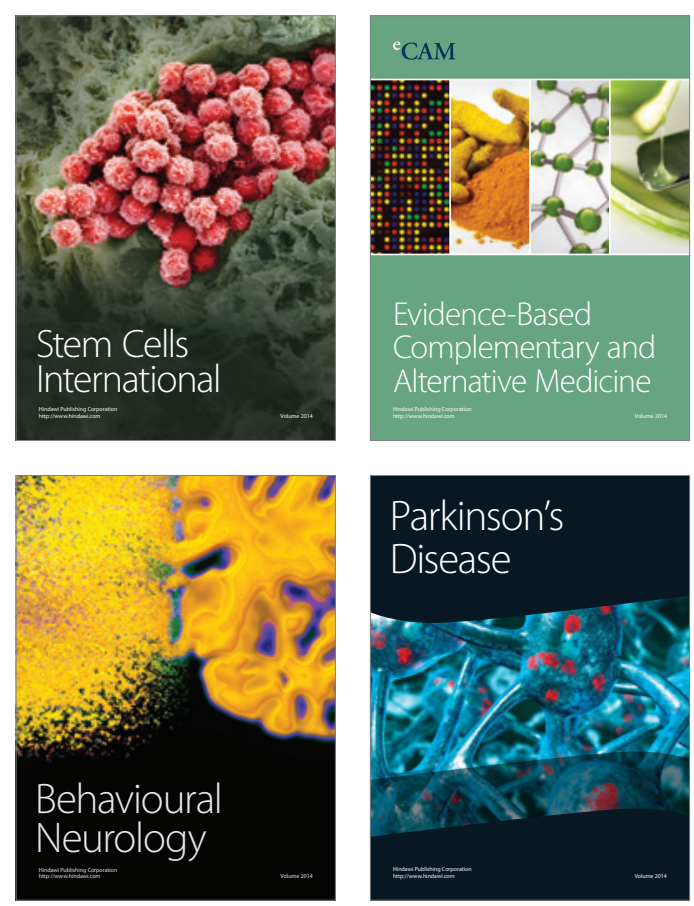

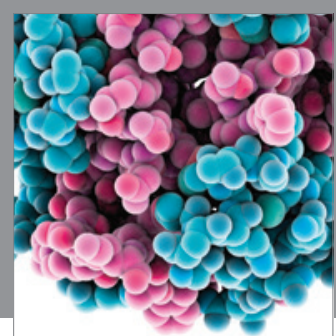

Journal of
Diabetes Research

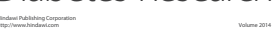

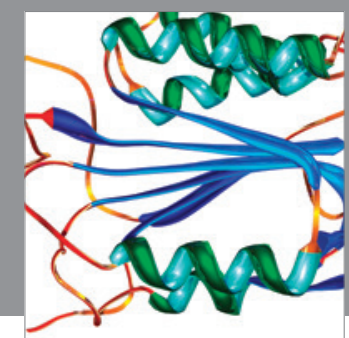

Disease Markers
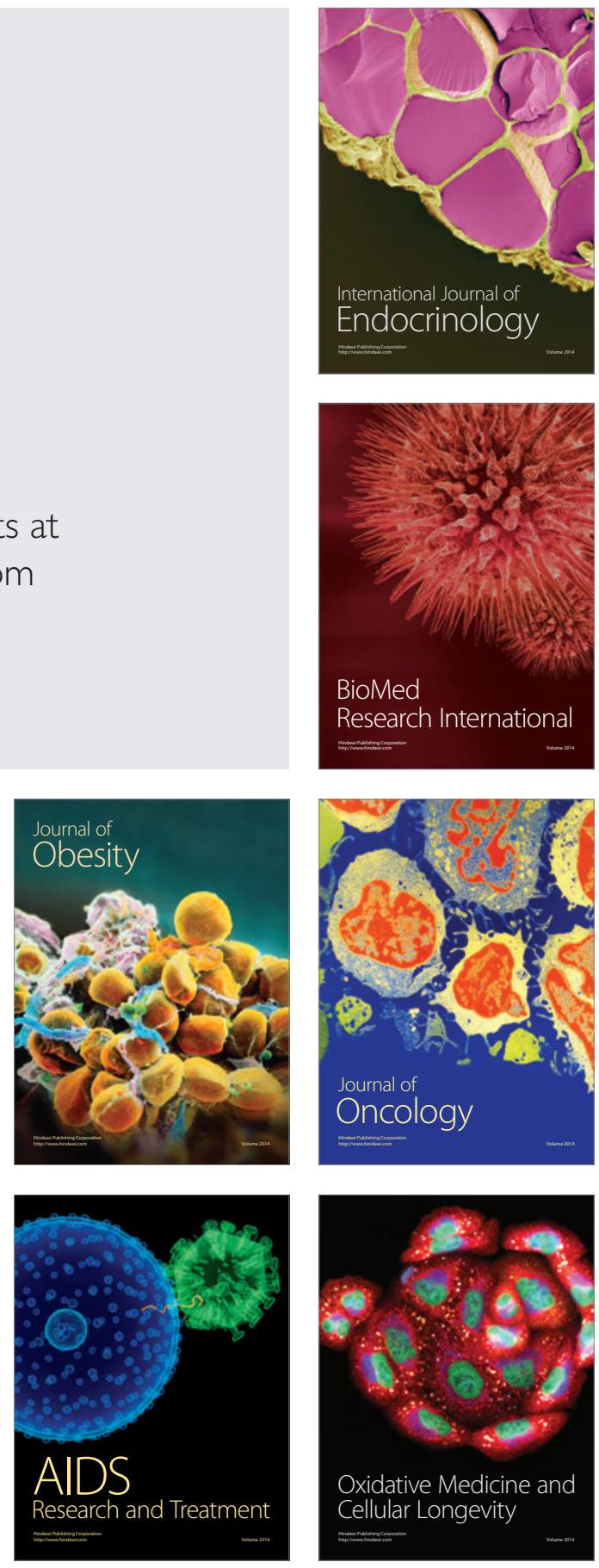